\title{
Design and development of an automated application for the analysis of monitoring data of Grid Connected Photovoltaic installations
}

\author{
D. Trillo-Montero, I. Santiago, J. Luna-Rodríguez and R. Real-Calvo \\ Department of Computer Architecture, Electronics and Electronic Technology \\ Córdoba University \\ Campus of Rabanales, 14071 Córdoba (Spain) \\ Phone: +0034 957 218699, /Fax number: +0034 957218373 \\ e-mail: ma2trmod@uco.es, el1sachi@uco.es, el1luroj@uco.es, el1recar@uco.es
}

\begin{abstract}
The aim of this paper was to design and develop an application for automating the analysis of the operating point of grid-connected photovoltaic (PV) installations. This application integrates data from all of the devices that monitor information on PV installations, such as environmental sensors, inverters and meters, and it automates the storage, treatment and easy access to all information. The application is comprised by a Relational Data Base Management System (RDBMS), with a common database to store the technical data of the various components of each PV installation, and a database for each of the installations to be analyzed, linked among them and with the previous one, where data from monitoring and calculated magnitudes would be stored. Moreover a software named S.lar was developed to determine, from monitored data, some standard magnitudes related to performances and losses of PV installation components at different time scales, and to visualize in a graphical and numerical way all the stored information. This tool enables to analyze PV facilities installed by small companies, with different configurations and technical specifications, in order to control and improve their production and maintenance, based on a more comprehensive and detailed study of their real operation.
\end{abstract}

\section{Key words}

Grid connected photovoltaic installations, Software development, Analysis of data

\section{Introduction}

Within the framework of renewable energy, solar photovoltaic energy (PVE) has been consolidated as the third largest source of renewable energy after hydro and wind, due to the important technological developments achieved and the increasing installed PV capacity, with more than $100 \mathrm{GW}$ in all the world [1].

One of the opportunities for progress in this sector is based on the improvement of the performance and the better use of existing technologies, which still function below their expected values. It will allow decrease the net costs that these facilities need, in order to improve their competitiveness with respect to other modes of power generation. If the installed power of these types of installations keeps increasing, a small improvement in performance percentage would lead to a significant net improvement in production.

The significant growth of grid-connected PV systems has not been accompanied by the implementation of resources for diagnosis, monitoring and fault detection at the PV facility, and at present, only a very small number of the many facilities currently in service undertake a detailed analysis of the functioning of their components, beyond that of measuring the total energy generated. Most plants operating today, especially those below $25 \mathrm{kWp}$ power, do so without any supervision mechanism, despite the great importance of having effective failure control [2].

An exhaustive supervision of the behavior of a PV plant requires, firstly, the presence of a system for monitoring the operating parameters of the various devices that comprise it. In the installations already in operation, most inverters themselves, aided by some additional sensors, can mainly perform this task without posing a significant additional economic cost in investment. However, such a monitoring system would also require an application to process easily and automatically the large volume of data recorded, a task that could not otherwise be addressed by the staff of the small companies engaged in PV plant installation or maintenance.

Therefore, the objective of this work was to design and develop an application for automating the analysis of the operating point of various grid-connected PV installations. The aim is that it could be used both by small companies with installations already in operation, in order to improve the real operation of the plant taking advantage of their existing equipment, and by scientific researchers, thanks to its versatility in obtaining relationships between parameters and magnitudes. 
Although other authors have already developed software applications to analyze parameters from PV installations [3-5], or several companies have already commercialized such type of software [6], what differentiates the application developed in this paper, however, is that it provides a greater amount of information when analyzing PV plants, not only the total power or energy generated by the PV installation or its PR value, integrating data from all of the devices that record information in the installation, such as environmental sensors, inverters and meters, and from several installations with different technical specifications, enabling a comparison among them.

\section{Methodology.}

An application to characterize grid-connected PV systems should have available, in the first place, the technical specifications of all photovoltaic system components of the installations to be studied (configurations, models and characteristics of modules, inverters and meters).

Secondly, parameters monitored in the PV installations must also be included in the application. These parameters are regulated by the European standard UNE-EN 61724:1998, "Monitoring of photovoltaic systems Guidelines for measurement, data exchange and analysis" [7]. However, in practice monitoring in PV systems already in operation is carried out via their inverter equipment, and not all inverter models monitor all of regulated parameters, and obviously each manufacturer or, indeed, each model can use a different notation for each of the parameters - even to the point of using different measurement units for the same magnitude.

All of this information, which must be recorded and stored in a database system, needs to be processed in order to obtained a series of standardized behaviour magnitudes and rates, which enables to analyze the performance and losses of each of the installation components. These magnitudes, which are calculated from monitored parameters such as irradiance, temperature, voltage, current, energy and power, as well as using the technical information of the plant, are Module Efficiency $\left(\eta_{\mathrm{G}}\right)$, Inverter Efficiency $\left(\eta_{\mathrm{INV}}\right)$ and System Efficiency $\left(\eta_{\mathrm{s}}\right)$; Reference Yield $\left(\mathrm{Y}_{\mathrm{r}}\right)$, Temperature Corrected Yield $\left(\mathrm{Y}_{\mathrm{t}}\right)$, Array Yield $\left(\mathrm{Y}_{\mathrm{A}}\right)$ and Final Yields $\left(\mathrm{Y}_{\mathrm{f}}\right)$; Thermal capture Losses $\left(\mathrm{L}_{\mathrm{ct}}\right)$, Miscelaneous capture losses $\left(\mathrm{L}_{\mathrm{cm}}\right)$, Capture losses $\left(\mathrm{L}_{\mathrm{c}}\right)$, and Inverter Losses $\left(\mathrm{L}_{\mathrm{s}}\right)$; and the Performance ratio (PR) [7]. They can be used to compare the performance between systems with different characteristics and geographic locations.

For a complete analysis, these magnitudes should be determined for different time scales. Firstly, "instantaneous values", which will have the same temporal frequency as monitoring parameters, must be calculated. It is also necessary to determine the so-called "accumulated or aggregated values" of these magnitudes. These must be calculated to produce an hourly scale (the sum of the instantaneous values recorded or calculated for each hour); a daily scale (sum of the 24-hourly values for each day) and a monthly scale (sum of the daily values for each month). It is also important to calculate the "monthly average values", which are determined by all of the daily average values in each month, as well as the maximum and minimum values for each parameter or quantity. Using all of this information, the PV system can be fully characterized.

All of this information was stored in a RDBMS developed in this work, which was comprised of a common database, to store the technical data of the various components of every PV installation, and of a database for each of the installations to be analyzed, linked among them and with the previous one, where data from monitoring and calculated magnitudes would be stored. The division of information into different databases was necessary, because if all this information were stored in a single database, a significant increase in memory and an increment in the processing and response time would result.

MySQL, a very popular management system, was used for the implemented database [8]. It was chosen because it is a flexible, scalable, high-performance, high-availability and robust system. Moreover it provides free and very complete support for the great majority of development environments, such as NET, Java or PHP, as well as providing a variety of free tools available for its administration, such as MySQL WorkBench or HeidiSQL. MySQL flexibility enabled us to establish relationships between fields common to tables in different databases and the creation of queries involving tables and queries from different databases.

As well as the RDBMS, a software application, called S.lar, was developed. This application enables monitoring data to be entered into different tables in the RDBMS, by automating the calculation and storage of the standard magnitudes and performance rates of each individual component of PV installations. C\# programming language was used to develop this application. To carry out the desktop application environment, a free version of Microsoft Visual C\# 2010 Express [9] was employed. It was also necessary to include free code (Open Source) and different free libraries.

\section{Results}

The software developed has a main menu, whose user interface is reflected in Figure 1, which includes, on a one hand, a series of buttons called Installations, Inverters, Meters, Parameters and Ma nufacturers. These buttons, whose functionality is linked to the tables in the common database, are associated with a set of forms that enable the technical specifications of the installation's components to be entered into the common database.

On the other hand, another set of buttons also appears in the main menu (Figure 1). The Data Migration button gives access to a form that automates the data migration 
process. Before migrating data, all of the information concerning the PV installation required by the common database must have been entered beforehand, using the forms provided for this purpose. In the programming of data migration process for S-lar software different migration paths were developed depending on the data type, in order for such data to be stored in separate tables. Moreover, the insertion mechanism was made flexible enough to be easily adaptable to monitoring data files with different internal structures (from different models of inverters and/or meters). It also took into account the fact that these files are usually stored in directories whose organization may differ from one installation to another.

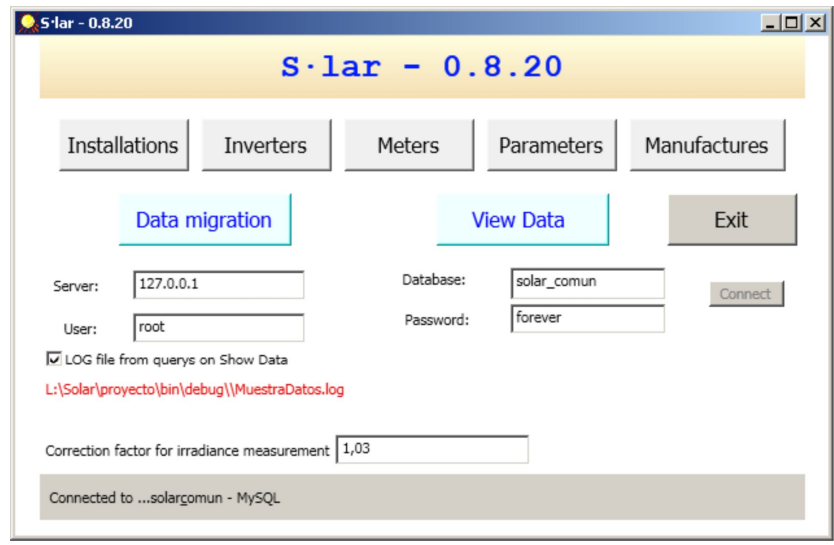

Fig. 1. S.lar software's main menu.

Once the migration process is completed, the next step is to calculate all of the standard magnitudes indicated in section 2. To this end, the $S$-lar application performs a series of mathematical operations on the monitored parameters, while also determining aggregate and average values. Depending on the inverter model, it can monitor different parameters. Therefore in S.lar programming the particularization of different routines for accessing to the calculation module was contemplated, selecting automatically in each case the correct parameters recorded by the inverter that will lead to the desired magnitude. For example, the energy generated by modules or obtained at an inverter's output can be calculated either through the voltage and current values, using the power values or directly from the monitored energy values. However, all of these parameters may or may not be included in the monitored data, depending on the inverter model installed in the PV plant.

And finally, the View Data button (Figure 1) gives access to a data visualization window, where data stored in databases are displayed either graphically or numerically. This window enables the user a fast, simple and intuitive access to all parameters and magnitudes stored in databases of every PV plant.

The $S$-lar data visualization window (Figure 2) possesses three main zones: Selection, in the upper; Detail, to the left, and Representation, which occupies a larger area in the center of the window.

In the Selection region there are a series of buttons and menus that enable the user to perform the functions described below. Firstly, there are three pull-down menus for selecting the name of the installation to be studied; how to display the selected data (numerically or graphically) and the type of parameters and magnitudes to be displayed (instantaneous, aggregated or average; from inverter or from meters), respectively. The selection of each type of data is associated with a certain type of query to be performed in the databases.

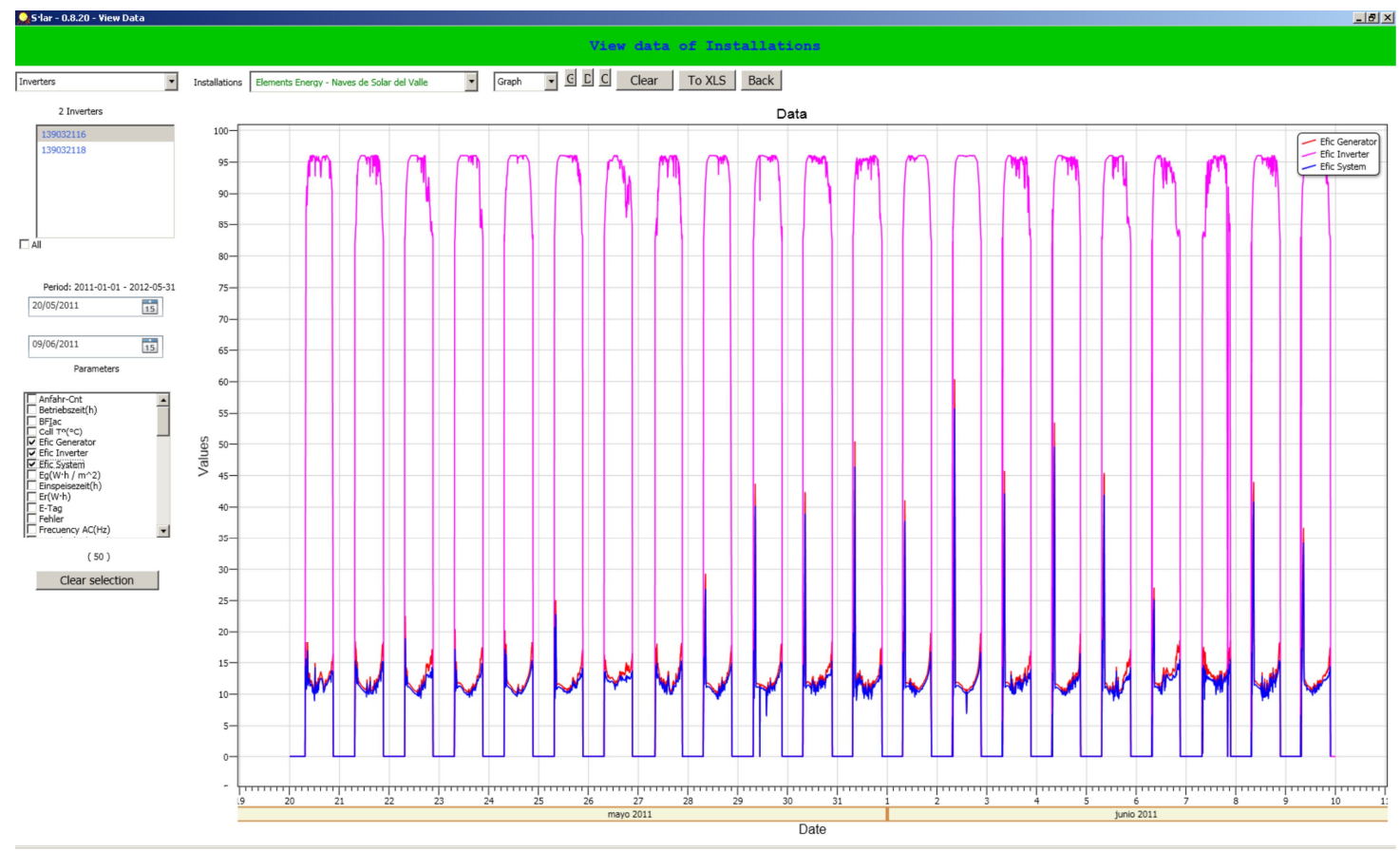

Fig. 2. S·lar software's display window with graphical representation of selected data. 
Secondly, there are other buttons that allow previously selected data to be exported to Microsoft Excel, with the aim of extending its analysis (Figure 2). To this end, there is the option of creating the export destination file or of selecting a previously-created file. By clicking on the To $X L S$ button, data selected previously appears in a new spreadsheet that will be created in the selected Excel file.

Finally, in this section of the window there is also a button to clear the selected data in the display window and a button to exit (Back).

In the Detail region there is a box at the top showing the inverter and meter codes which will correspond to the PV installation previously selected in the option available in the Selection region. This box enables the user to select the inverter or the meter whose data we want to work with. Just below, there is an option for selecting the time interval for which we want to display the data for the chosen inverter or meter. At the bottom there is another box where the user can select one or more parameters or magnitudes to be visualized. As many parameters as desired of the same inverter or the same meter can be selected. The software has also the ability to select a single magnitude or parameter and display it for all of the inverters belonging to the same installation. This option is useful for comparing the behavior of individual inverters or their associated modules. The button just below (Clear selection) enables the user to remove the selection of parameters or magnitudes previously performed.

Finally, in the Representation area, previously selected parameters or variables can be visualized both, graphically over time, or numerically in tabular form. An example of the first option is reflected in Figure 2 where the instantaneous efficiency data of one inverter, of its associated PV modules and of the system formed by both in a particular installation, are plotted as a function of time for a total of 21 days in May and June 2011. Due to the optimization of the application's response time, the information requested is obtained in both cases almost immediately, despite the high volume of information with which databases work.

As an example of using the application developed in this work, a sample of some of the results obtained after analyzing the monitoring data from a particular grid connected PV system, located in Cordoba (Spain), is shown in Figures 3, 4 and 5. This installation has three inverters, and each one receives the energy generated by 36 photovoltaic modules, each one with a nominal power of $165 \mathrm{~W}$. In these figures the daily average PR values versus the total daily irradiance of each inverter during 2011 are exposed, magnitudes easily obtained making use of S.lar data visualization window, after a few simple mouse clicks. In addition to the relationship between these two magnitudes, which reflects that when the total daily irradiance grows the installations are more efficient, a remarkable aspect after seeing these figures is that the system formed by the inverter 3 , together with the modules associated with it, are operating with a worse performance than the other two inverters.

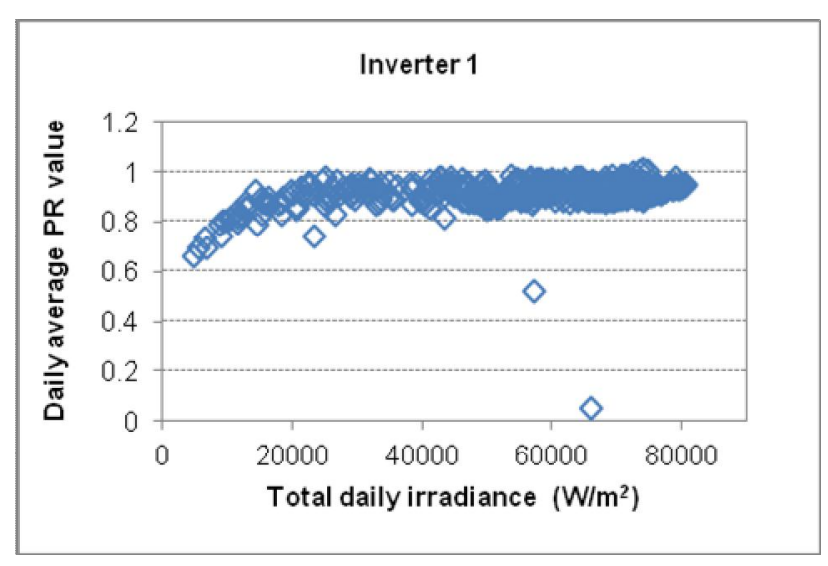

Fig. 3. Daily average PR values versus total daily irradiance, of inverter 1, from January to December 2011.

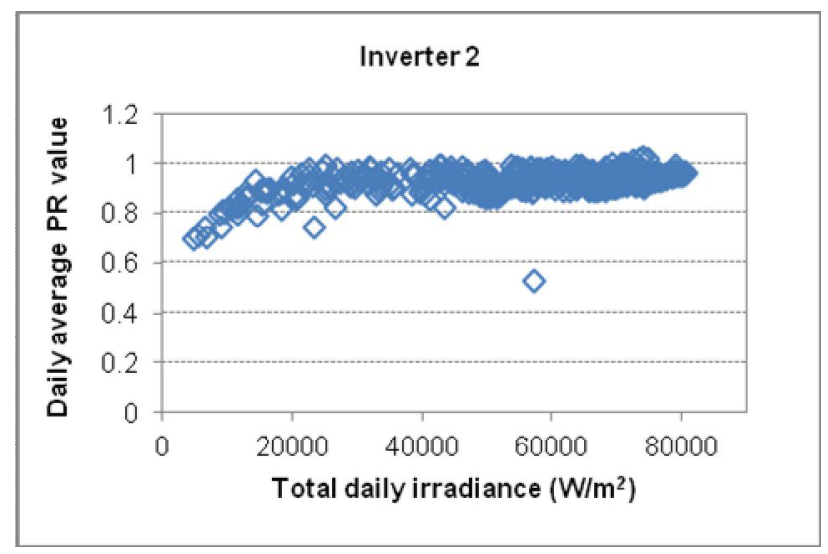

Fig. 4. Daily average PR values versus total daily irradiance, of inverter 2, from January to December 2011.

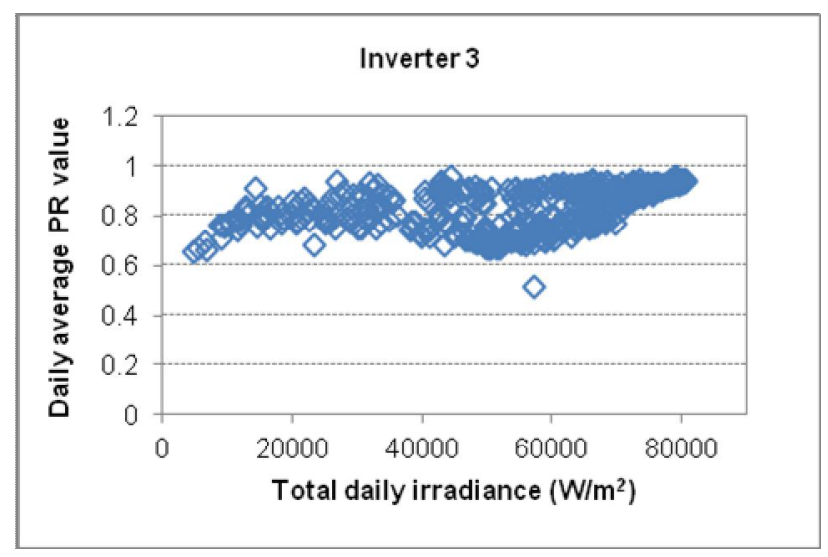

Fig. 5. Daily average PR values versus total daily irradiance, of inverter 3, from January to December 2011.

To find the causes of this, the efficiencies of the three inverters as well as that of their associated modules, were analyzed. The average efficiency value for the 508 days analyzed, easily obtained by the use of S.lar application, was $94.27,94.39$ and $94.38 \%$ for each of the three inverters respectively, indicating that they all operated with the same degree of efficiency. However the efficiency of the modules associated with the third inverter decreased during the winter months, having on 
average values approximately $15 \%$ less than those of the other two. To located and corrected the reasons for better future performance, the average daily values of the final yield $\left(Y_{f}\right)$, losses in modules (due to temperature, $L_{c t}$, and the miscellaneous losses, $\left.L_{c m}\right)$ and inverter losses $\left(L_{s}\right)$ for inverters 1 and 3 were obtained in January of 2012. Temperature losses $\left(L_{c t}\right)$ were 6.2 and $4.8 \%$ of the production in inverters 1 and 3 respectively, while inverter losses $\left(L_{s}\right)$ were $6.5 \%$ of the production in both inverters. However miscellaneous losses corresponded to 2.6 and $29.2 \%$ of production respectively for inverters 1 and 3 (Figures 6 and 7), being the presence of shadows in winter months the cause of these very higher losses in production in the third inverter.

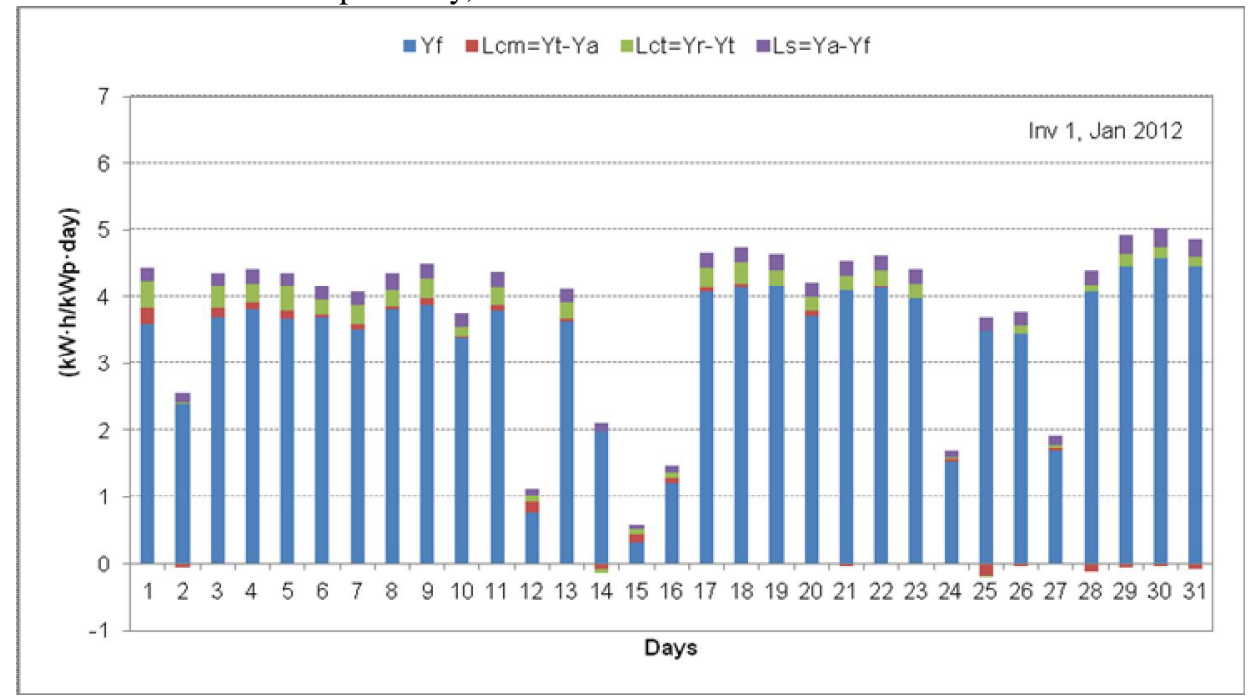

Fig. 6. Average daily values of final yield $\left(Y_{f}\right)$, losses in the modules (due to temperature, $L_{c t}$, and miscellaneous losses, $L_{c m}$ ) and losses in the inverters $\left(L_{s}\right)$, for inverter 1 in January 2012.

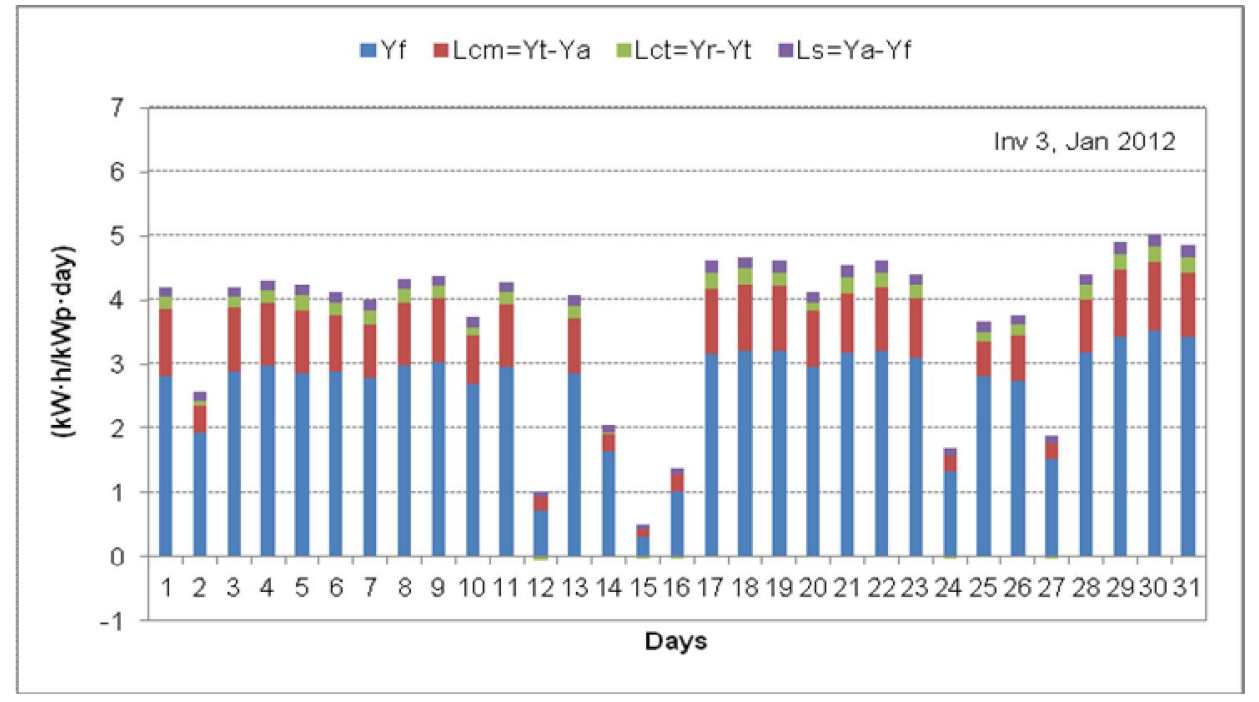

Fig. 7. Average daily values of final yield $\left(Y_{f}\right)$, losses in the modules (due to temperature, $L_{c t}$, and miscellaneous losses, $L_{c m}$ ) and losses in the inverters $\left(L_{s}\right)$, for inverter 3 in January 2012.

\section{Conclusions}

In this work an application for automating the analysis of the operating point of grid-connected photovoltaic (PV) plants was designed and developed. The application developed integrates data from all of the devices that record information on PV installations, such as environmental sensors, inverters and meters. Moreover, it is versatile enough for it to be adapted to different types and models of inverters, the devices which, generally, are responsible for monitoring most of the facilities installed by small companies. This application provides an useful and intuitive tool which turns into an easy task the treatment of the large volume of information recorded in each plant.

This application provides the values of all standardized performance and loss indexes of each PV plant components, calculated in different time scales, which can allow small installers have information available, apart from the mere energy injected into the grid, which can help them for the maintenance and optimal operation of the present and future installations. In addition, the application enables to establish relationships between 
parameters very easily, which allows it to be used also at research level.

As it can be indicated the applications are comprised by a RDBMS. It provides an orderly, accessible and fast storage system, with no space limitation, in order to store and provide users with all of the information from PV systems (each of the technical specifications of the components for the grid-connected photovoltaic systems, and the value of all monitored parameters and calculated magnitudes). The access and loaded of this RDBMS is controlled by the implemented S.lar software, which automates the calculation and storage of the standard magnitudes and performance rates of each individual component of PV installations. Moreover, making use of intuitive menus and forms, it allows user to visualize numerical and graphically all the stored information.

Although the developed application works off-line, once data have been recorded, it would be interesting to adapt the application so that it could work online in real time and to develop a web application available for PCs and mobile devices. These improvements are already being implemented and they will be discussed in a future paper.

\section{Acknowledgement}

This work was supported in part by the agreement established between the company Solar del Valle SL, and the University of Córdoba, Spain.

\section{References}

[1] European Photovoltaic Industry Association (EPIA), http://www.epia.org/

[2] Chouder A, Silvestre S. "Automatic supervision and fault detection of PV systems based on power losses analysis". Energy Conversion and Management 2010, 51, pp. 19291937.

[3] Tsankov P. Software for monitoring a grid-connected photovoltaic power plant. Contemporary Materials (Renewable energy sources) 2011, 2 (2), pp. 191- 203.

[4] Fernández-Pacheco DG, Molina-Martínez JM, Ruiz-Canales A, Jiménez M. A new mobile application for maintenance tasks in photovoltaic installations by using GPS data. Energy Conversion and Management 2012, 57, pp. 79-85.

[5] Gagliarducci M, Lampasi DA, Podesta L. GSM-based monitoring and control of photovoltaic power generation. Measurement 2007, 40, pp. 314-321.

[6] SMA. http://www.sma.de/en.html www.smaiberica.com/ Consultado por última vez: 25 June 2013.

[7] European Standard UNE-EN 61724:1998, Photovoltaic system performance monitoring - Guidelines for measurement, data exchange and analysis, 1998.

[8] MySQL. www.mysql.com

[9] Microsoft Visual C\# Express 2010. http://www.microsoft.com/visualstudio/eng/products/v isual-studio-2010-express 